\title{
On the radial distribution function of a hard-sphere fluid
}

\author{
M. López de $\operatorname{Har}{ }^{*}$ \\ Centro de Investigación en Energía, U.N.A.M., Temixco, Morelos 62580, Mexico \\ A. Santo: \\ Departamento de Física, Universidad de Extremadura, E-06071 Badajoz, Spain
}

\begin{abstract}
Two related approaches, one fairly recent [A. Trokhymchuk et al. J. Chem. Phys. 123, 024501 (2005)] and the other one introduced fifteen years ago [S. B. Yuste and A. Santos, Phys. Rev. A 43, 5418 (1991)], for the derivation of analytical forms of the radial distribution function of a fluid of hard spheres are compared. While they share similar starting philosophy, the first one involves the determination of eleven parameters while the second is a simple extension of the solution of the Percus-Yevick equation. It is found that the second approach has a better global accuracy and the further asset of counting already with a successful generalization to mixtures of hard spheres and other related systems.
\end{abstract}

The fluid of hard spheres (HS) is a key model in the use and application of statistical mechanics to the study of the thermodynamic and structural properties of real fluids. This is due not only to the relative simplicity of the intermolecular potential but also to the availability of rather accurate (albeit semi-empirical) expressions for the corresponding equation of state (EOS) $\frac{1}{1}$ as well as the exact solution of the Ornstein-Zernike equation with the Percus-Yevick (PY) closure ${ }_{2}^{2}$ that provides an approximate analytical expression for the radial distribution function (rdf). Yet, interest in this system is very much alive since exact analytical expressions both for its thermodynamic and structural properties have defied derivation. A recent example of this interest of particular relevance to our purpose here is the analytical expression for the rdf proposed by Trokhymchuk et al. (TNJH) $?^{3}$

Fifteen years ago, two of us $\frac{4}{\underline{\underline{4}}}$ developed an analytical method to determine reliable expressions for the rdf $g(r)$ of an HS fluid. Such method provides a reasonable extension of the solution to the PY equation by imposing weak physical requirements. Apart from being relatively simple, requiring as the only input the EOS of the HS fluid, it has the further asset of including by construction the full thermodynamic consistence between the virial and compressibility routes to the EOS. The method uses a rational function ansatz for a function related to the Laplace transform of $r g(r)$, and so it is referred to as the Rational Function Approximation (RFA) method. It has subsequently been used to study the metastable region past the liquid-solid transition,,$\frac{5}{5}$ successfully adapted and generalized to deal with other systems (such as HS mixtures,$\underline{6}$ sticky hard spheres $\stackrel{7}{\underline{7}}$ and square-well fluids ${ }^{8}$ ), used to derive other structural properties (such as the contact values of the derivatives of the rdf, the direct correlation function, and the bridge function ${ }^{9}$ ) and also in connection with the perturbation theory of liquids $\underline{10}$

Interestingly enough, there are several common features in the RFA method and in the TNJH approach ${ }^{3}$ to derive an approximate analytical form for $g(r)$. For instance, the underlying philosophy is the same as evidenced through the following text that we quote from
Ref. 3: "A third possibility towards obtaining a nonempirical analytic representation of $g(r)$ is to assume a certain theoretically justified functional form for $g(r)$ (e.g., a result of the solution of the $\mathrm{OZ}$ equation) with adjustable parameters and impose then certain constraints (e.g., thermodynamic consistency) to determine these parameters." Such a statement is totally applicable to the RFA method. Furthermore, both use the asymptotic behavior of the rdf for $r \rightarrow \infty$, they enforce thermodynamic consistency, and both also require as input only a prescribed compressibility factor $Z \equiv p / \rho k_{B} T$ and its associated isothermal susceptibility $\chi=[\partial(\rho Z) / \partial \rho]^{-1}$. Therefore it seems natural to compare the outcomes of both approaches in order to assess their merits and limitations. The purpose of this Note is to carry out such a comparison.

In the TNJH approach,$\frac{3}{,}$ the $\mathrm{rdf}$ is written as

$$
g(r)= \begin{cases}g_{\mathrm{d}}(r), & 1<r<r_{\mathrm{m}} \\ g_{\mathrm{s}}(r), & r>r_{\mathrm{m}},\end{cases}
$$

where $r_{\mathrm{m}}\left(1<r_{\mathrm{m}}<2\right)$ denotes the first minimum of $g(r)$ and the "depletion" (d) and "structural" (s) parts have the forms

$$
\begin{gathered}
\left.g_{\mathrm{d}}(r)=\frac{A}{r} e^{\mu(r-1)}+\frac{B}{r} \cos (\beta(r-1)+\gamma)\right) e^{\alpha(r-1)}, \\
g_{\mathrm{s}}(r)=1+\frac{C}{r} \cos (\omega r+\delta) e^{-\kappa r},
\end{gathered}
$$

respectively. In Eqs. (11)-(3) and henceforth units are taken in which the diameter $\sigma$ has a value of 1 . The depletion and structural parts have the same functional dependence on $r$ as the exact solution of the PY equation in the shell $1<r<2$ and in the limit $r \rightarrow \infty$, respectively. The approximation (1) contains eleven parameters to be determined as functions of the density $\rho$ (or, equivalently, of the packing fraction $\eta \equiv \pi \rho \sigma^{3} / 6$ ). Trokhymchuk et al. impose five natural conditions: consistency with prescribed $Z$ and $\chi$, continuity of $g(r)$ and 
its first derivative at $r=r_{\mathrm{m}}$, and the minimum condition at $r=r_{\mathrm{m}}$. More explicitly,

$$
\begin{gathered}
g\left(1^{+}\right)=\frac{Z-1}{4 \eta}, \quad \int_{0}^{\infty} d r r^{2}[g(r)-1]=\frac{\chi-1}{24 \eta} \\
g_{\mathrm{d}}\left(r_{\mathrm{m}}\right)=g_{\mathrm{s}}\left(r_{\mathrm{m}}\right),\left.\quad \frac{\partial g_{\mathrm{d}}(r)}{\partial r}\right|_{r_{\mathrm{m}}}=0,\left.\quad \frac{\partial g_{\mathrm{s}}(r)}{\partial r}\right|_{r_{\mathrm{m}}}=0
\end{gathered}
$$

In Ref. 3 the Carnahan-Starling-Kolafa (CSK) ${ }^{11}$ EOS is used for $Z$ and $\chi$. Furthermore, the parameters $\gamma$ and $\mu$ are fixed at their PY expressions ${ }^{2} \kappa$ and $\omega$ being given by parameterizations obtained by Roth et al ${ }^{12}$ This still leaves seven parameters to be determined under the five constraints (4) and (5). By using the first equation of (4) and the first and third equations of (5), $A, B, C$, and $\delta$ are expressed in terms of $\alpha, \beta$, and $r_{\mathrm{m}}$, as well as of $g_{\mathrm{m}} \equiv g\left(r_{\mathrm{m}}\right)$. These remaining four unknowns are determined by applying two conditions, namely, the second equation of (4) and the second equation of (5), and "by minimizing an appropriate functional," the form of which is not given in Ref. 33. In any case, due to the highly nonlinear character of the problem, the numerical solutions for $\alpha, \beta, r_{\mathrm{m}}$, and $g_{\mathrm{m}}$ are parameterized as functions of $\eta$ in the range $0.2 \leq \rho \sigma^{3} \leq 0.9$.

On a different vein, in the RFA method the Laplace transform of $r g(r)$ is taken to be given by

$$
G(t)=\mathcal{L}\{r g(r)\}=\frac{t}{12 \eta} \frac{1}{1-e^{t} \Phi(t)},
$$

where

$\Phi(t)=\left(1+S_{1} t+S_{2} t^{2}+S_{3} t^{3}+S_{4} t^{4}\right) /\left(1+L_{1} t+L_{2} t^{2}\right)$,

the six coefficients $S_{1}, S_{2}, S_{3}, S_{4}, L_{1}$, and $L_{2}$ (which depend on the packing fraction) being evaluated in an algebraic form by imposing the following requirements $:^{4.5}$ (i) $\chi$ must be finite and hence the first two integral moments of the total correlation function $h(r) \equiv g(r)-1$, i.e., $\int_{0}^{\infty} d r r^{n} h(r)$ with $n=1,2$, must be well defined; (ii) the approximation must be thermodynamically consistent with a prescribed EOS, i.e., Eq. (4) must be verified. Using the first requirement one finds that $L_{1}, S_{1}$, $S_{2}$, and $S_{3}$ are linear functions of $L_{2}$ and $S_{4}$. The choice $L_{2}=S_{4}=0$ yields the PY solution ${ }^{2}$ On the other hand, imposing the requirement (ii) leads ${ }^{4.5}$ to explicit expressions for $L_{2}$ and $S_{4}$ in terms of $\chi$ and $Z$. In turn, the rdf is given by

$$
g(r)=\frac{1}{12 \eta r} \sum_{n=1}^{\infty} \varphi_{n}(r-n) \Theta(r-n),
$$

with $\Theta(r)$ the Heaviside step function and $\varphi_{n}(r)=$ $-\mathcal{L}^{-1}\left\{t[\Phi(t)]^{-n}\right\}$, which can be explicitly expressed by using the residue theorem ${ }^{\frac{5}{*}}$ To close the problem one has to give an EOS to fix $Z$ and $\chi$, so all the procedure is a function of this choice. Here, we take the same choice as in Ref. 3, namely the CSK EOS.11
The form of the TNJH approximation, Eqs. (11)-(3), is particularly simple, while the RFA method, as happens with the PY solution, gives an explicit expression (7) for each separate shell $n<r<n+1$. On the other hand, the formal simplicity of the TNJH is at the expense of introducing a number of unknowns exceeding the number of constraints, so that minimization of a certain empirical functional is needed. As a consequence, the problem becomes highly nonlinear and the numerical solutions are fitted to given expressions; a change in the desired EOS would then require to start all over again the fitting procedure. In contrast, the RFA only requires six coefficients which are explicitly expressed in terms of an arbitrary EOS. From a less practical but more fundamental point of view, it can be added that the TNJH $g(r)$ introduces an artificial second-order discontinuity at the minimum $r=r_{\mathrm{m}}$, while it forces $g(r)$ to be analytic at $r=2,3, \ldots$

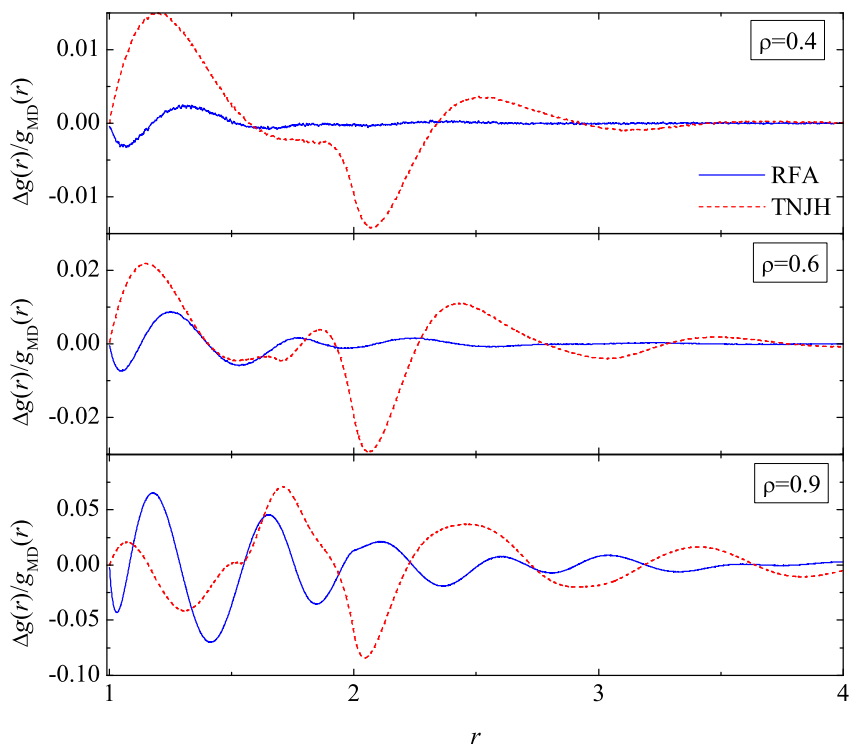

FIG. 1: (Color online) Relative deviation $\Delta g(r) / g_{\mathrm{MD}}(r) \equiv$ $\left[g(r)-g_{\mathrm{MD}}(r)\right] / g_{\mathrm{MD}}(r)$ of the values of the rdf of an HS fluid with respect to the molecular dynamics simulation data, ${ }^{13}$ $g_{\mathrm{MD}}(r)$, as a function of $r$ for $\rho \sigma^{3}=0.4$ (top panel), $\rho \sigma^{3}=0.6$ (middle panel), and $\rho \sigma^{3}=0.9$ (bottom panel). The dashed lines represent the results of the approach of Ref. 3, while the solid lines refer to those of the RFA method. ${ }^{4}$

We are now in a position to carry out the aforementioned comparison. Since both methods lead to reasonably accurate values for the rdf, in Fig. 11 we present the results of the relative deviations of such values with respect to the very recent simulation data 13 for three characteristic densities. It is quite clear that only in the case $\rho \sigma^{3}=0.9$ and for small distances, the RFA values give a somewhat poorer agreement with the simulation results. In any case, and recognizing the value of the TNJH approach, ${ }^{3}$ it is fair to say that the RFA method provides a rather accurate and simple alternative to compute the structural properties of the HS fluid. Certainly, 
there are fewer parameters in the RFA method and they have a clear physical link. Moreover, as the authors of Ref. 3 point out, the availability of analytical results in instances where simulations may fail (such as HS mixtures of a large size ratio) is crucial. In this context, the fact that we already have a simple extension of our approach to deal successfully with these and related systems 6.7 .8 indicates that the RFA method is a valuable tool for the theoretical investigation of the structural properties of hard-core systems.

\section{Acknowledgments}

We are grateful to J. Kolafa for kindly providing us with tables of computer simulation results. Thanks are also due to I. Nezbeda and A. Trokhymchuk for an exchange of electronic correspondence that helped us to clarify some points and understand better their formulation. The research of A.S. and S.B.Y. has been supported by the Ministerio de Educación y Ciencia (Spain) through grant No. FIS2004-01399 (partially financed by FEDER funds) and by the European Community's Human Potential Programme under contract HPRN-CT-2002-00307, DYGLAGEMEM.
* Electronic address: malopez@servidor.unam.mx URL: http://cie.unam.mx/xml/tc/ft/mlh/

$\dagger$ Electronic address: andres@unex.es URL: http://www.unex.es/eweb/fisteor/andres/

$\ddagger$ Electronic address: santos@unex.es URL: http://www.unex.es/eweb/fisteor/santos/

1 A few notable examples are listed in Table 1 of A. Mulero, C. Galán, and F. Cuadros, Phys. Chem. Chem. Phys. 3, 4991 (2001).

2 M. S. Wertheim, Phys. Rev. Lett. 10, 321 (1963); E. Thiele, J. Chem. Phys. 39, 474 (1963).

3 A. Trokhymchuk, I. Nezbeda, J. Jirsák, and D. Henderson, J. Chem. Phys. 123, 024501 (2005); Erratum: ibid. 124, 149902 (2006).

4 S. B. Yuste and A. Santos, Phys. Rev. A 43, 5418 (1991). In the case of a monodisperse HS fluid, the RFA method yields results equivalent to the generalized mean spherical approximation worked out by E. Waisman, Mol. Phys. 25, 45 (1973).

5 S. B. Yuste, M. López de Haro, and A. Santos, Phys. Rev. E 53, 4820 (1996); M. Robles, M. López de Haro, A. Santos, and S. B. Yuste J. Chem. Phys. 108, 1290 (1998); M. Robles and M. López de Haro, Europhys. Lett. 62, 56 (2003); J. Phys.: Condens. Matt. 16, S2089 (2004).

6 S. B. Yuste, M. López de Haro, and A. Santos, J. Chem. Phys. 108, 3683 (1998); Al. Malijevský, A. Malijevský, S.
B. Yuste, A. Santos, and M. López de Haro, Phys. Rev. E 66, 061203 (2002).

7 S. B. Yuste and A. Santos, J. Stat. Phys. 72, 703 (1993); Phys. Rev. E 48, 4599 (1993); A. Santos, S. B. Yuste, and M. López de Haro, J. Chem. Phys. 109, 6814 (1998).

8 S. B. Yuste and A. Santos, J. Chem. Phys. 101, 2355 (1994); L. Acedo and A. Santos, J. Chem. Phys. 115, 2805 (2001); J. Largo, J. R. Solana, L. Acedo, and A. Santos, Mol. Phys. 101, 2981 (2003); J. Largo, J. R. Solana, S. B.Yuste, and A. Santos, J. Chem. Phys. 122, 084510 (2005).

9 M. Robles and M. López de Haro, J. Chem. Phys. 107, 4648 (1997); S. B. Yuste, A. Santos, and M. López de Haro, Mol. Phys. 98439 (2000).

10 M. Robles and M. López de Haro, Phys. Chem. Chem. Phys. 3, 5528 (2001).

11 J. Kolafa, unpublished results (1986). This EOS first appeared as Eq. (4.46) in the review paper by T. Boublik and I. Nezbeda, Coll. Czech. Chem. Commun. 51, 2301 (1986).

12 R. Roth, R. Evans, and S. Dietrich, Phys. Rev. E 62, 5360 (2000).

13 J. Kolafa, S. Labík, and A. Malijevský, Phys. Chem. Chem. Phys. 6, 2335 (2004). See also http://www.vscht.cz/fch/software/hsmd/ for molecular dynamics results of $g(r)$. 